\title{
Charge-carrier properties in synthetic single-crystal diamond measured with the transient-current technique
}

\author{
H. Pernegger, ${ }^{\text {a) }}$ S. Roe, and P. Weilhammer ${ }^{\text {b) }}$ \\ CERN, Department of Physics, Geneva CH-1211, Switzerland \\ V. Eremin \\ Ioffe Physico-Technical Institute, Russian Academy of Science, Saint Petersburg 194021, Russia \\ H. Frais-Kölbl and E. Griesmayer \\ Fachhochschule für Wirtschaft und Technik, A-2700 Wiener Neustadt, Austria \\ H. Kagan \\ Department of Physics, Ohio State University, Columbus, Ohio 43210 \\ S. Schnetzer and R. Stone \\ Department of Physics and Astronomy, Rutgers University, New Jersey 08854 \\ W. Trischuk \\ Department of Physics, University of Toronto, Toronto M55 1A7, Canada \\ D. Twitchen and A. Whitehead \\ Element Six Ltd., King's Ride Park, Ascot, Berkshire SL5 8BP United Kingdom
}

(Received 9 July 2004; accepted 7 January 2005; published online 21 March 2005)

\begin{abstract}
For optimal operation of chemical-vapor deposition (CVD) diamonds as charged particle detectors it is important to have a detailed understanding of the charge-carrier transport mechanism. This includes the determination of electron and hole drift velocities as a function of electric field, charge carrier lifetimes, as well as effective concentration of space charge in the detector bulk. We use the transient-current technique, which allows a direct determination of these parameters in a single measurement, to investigate the charge-carrier properties in a sample of single-crystal CVD diamond. The method is based on the injection of charge using an $\alpha$ source close to the surface and measuring the induced current in the detector electrodes as a function of time. () 2005 American Institute of Physics. [DOI: 10.1063/1.1863417]
\end{abstract}

\section{INTRODUCTION}

The transient-current technique (TCT) is based on the direct measurement of the current pulse shape, which is induced in the electrodes of a detector by the drift of free charge carriers in an electric field. The duration of the current pulse equals the time the ionization charge needs to traverse the detector if the electric field is uniform and the charge lifetime is longer than the transit time. This technique relies on charge injection limited to a very small depth from the surface of the detector. From the transit time we determine the drift velocity and mobility. The TCT method has been used in the determination of charge-carrier mobility and velocity in insulators ${ }^{1}$ [CVD (chemical vapor deposition) diamond can be considered as an insulator] and, more recently, in the characterization of silicon $p-n$ junction detectors. ${ }^{2-4}$

The scope of this work is to extend the method to the characterization of single-crystal CVD diamonds and to show how it can be used beyond the determination of chargecarrier velocity and mobility over a wide range of electricfield strength. We also show that the precision measurement of the current pulse shape can be used as a direct measure-

\footnotetext{
a) Author to whom correspondence should be addressed; electronic mail heinz.pernegger@cern.ch

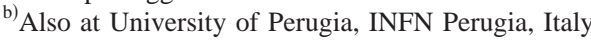

ment of electron and hole charge lifetimes and, if present in the detector, the effective space-charge concentration in the detector bulk. Our investigation is focused on demonstrating that the transient-current technique using $\alpha$ particles can be meaningfully applied to diamond samples, which are commonly used as high-speed and radiation hard particle detectors. We measure the charge-carrier properties of one singlecrystal diamond in a field strength range which is typically used for their operation $(0.2-1.5 \mathrm{~V} / \mu \mathrm{m})$ in tracking devices in particle physics experiments.

\section{CVD DIAMOND SAMPLE AND EXPERIMENTAL TECHNIQUE}

CVD diamonds have been investigated for several years as charged particle detectors in high radiation environments of particle physics experiments at the Large Hadron Collider, CERN, ${ }^{5}$ other accelerator-based particle physics experiments around the world, and are currently in use as radiation beam monitors. In high radiation environments CVD diamond benefits from its low generation leakage current due to its high band gap of $5.5 \mathrm{eV}$, and its fast current signal as a result of high charge-carrier mobility and limited charge lifetime. Additionally it can be manufactured in films of several hundred micrometer thickness, which allows the construction of a freestanding detector close to the interaction region. While 


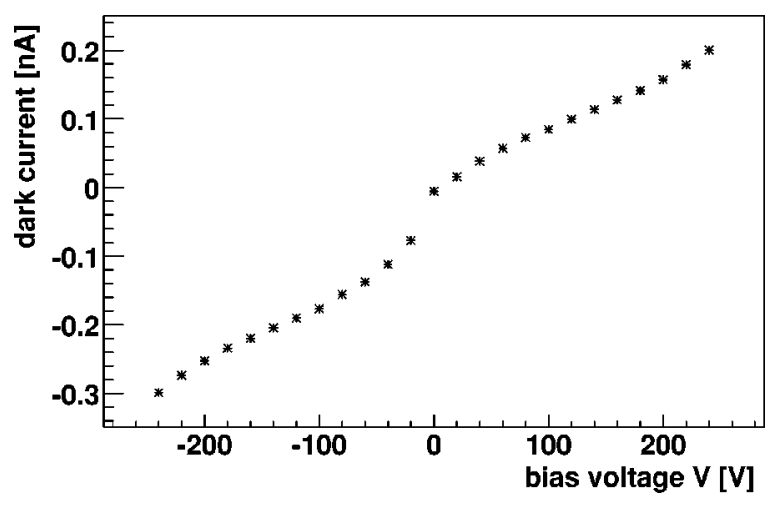

FIG. 1. Diamond dark current as a function of applied voltage.

most development to date has been performed on polycrystalline CVD diamond samples, recent measurements have shown that single-crystal CVD diamond films have superior electrical properties. The very low defect and dislocation densities present in highest-quality single-crystal diamonds lead to a significant improvement of electrical properties like mobility and charge lifetime. ${ }^{6}$

\section{A. Experimental setup}

The single-crystal diamond sample used in this study was synthesized with a microwave plasma-assisted CVD reactor and has been provided by the company Element Six Ltd.. ${ }^{7}$ The sample measures $4 \times 4 \mathrm{~mm}^{2}$ and is $470 \mu \mathrm{m}$ thick. Circular contacts are put on the top and bottom surfaces of the substrate. The contacts are $\mathrm{Cr}-\mathrm{Au}$ contacts with a total thickness of $250 \mathrm{~nm}$, which allow wirebond connections to the amplifier. The contacts do not include a guard ring. The top electrode is used as contact for the amplifier and the bottom electrode is used as contact to the externally supplied bias voltage. Before the sample has been used for the reported tests, the charge collection distance of the sample has been measured to be $470 \mu \mathrm{m}$ at an applied field of $1 \mathrm{~V} / \mu \mathrm{m}$, indicating a nearly full collection of generated ionization charge. ${ }^{8}$ The $I-V$ characteristics of the sample is shown in Fig. 1. The plot gives the sample's dark current, measured on a Keithley 237 source-measure unit, as a function of the applied voltage to the bottom contact. The shown data points in Fig. 1 were taken in the order of 0 to $240 \mathrm{~V}$ and 0 to $-240 \mathrm{~V}$; the data point shown at $0 \mathrm{~V}$ is the average of both measurements. The top contact is connected to the ground. Besides an equipment-related offset we observe a steady increase of dark current, including for low voltages below $\pm 100 \mathrm{~V}$. Within our measurement voltage range, the dark current does not saturate. Furthermore the sample has been characterized by cathodoluminescence studies, which showed a free exciton peak $(235 \mathrm{~nm})$ at $77 \mathrm{~K}$. Absorption measurements have been carried out and show the nitrogen level to be below detection limits; luminescence techniques show a small peak at $575 \mathrm{~nm}$, hence indicating the presence of a nitrogen vacancy complex, but at a concentration well below the detection limits of absorption techniques. Prior to metallization the bare diamond was polished by the manufacturer to obtain a smooth diamond surface. There are some indications, although not yet well established, that some methods of polishing introduce subsurface damage which alter the properties of the diamond to a depth of a few micrometers. As our measurement relies on the average drift of charges through the entire bulk, the possible effect of subsurface damage on the measured transit time is small, if any. Further investigations are currently underway to study the surface property of single-crystal diamonds using the transient-current technique.

For charge injection a collimated ${ }^{241} \mathrm{Am} \alpha$ source is mounted in the center of the top electrode at a distance of 1 $\mathrm{mm}$. The source was collimated using a screening foil between the electrode and the source with a hole of $1.5-\mathrm{mm}$ diameter. The sample is glued to a printed circuit board (PCB) which provides the bias voltage to the back contact and the connection to the external current amplifier. The entire assembly is mounted in a shielded and light tight metal box and operated at room temperature. For the measurement of hole signals a negative voltage is applied to the back contact; for the measurement of electron signals a positive voltage is applied.

In the separate measurement of electron and hole signals it is important that the deposited ionization charge can be regarded as a thin charge layer generated close to one electrode which subsequently drifts through the detector bulk. This is approximated in our setup by the range of ${ }^{241} \mathrm{Am} \alpha$ particles of $14 \mu \mathrm{m}$, which is small compared to the sample thickness of $d=470 \mu \mathrm{m}$. In order to avoid polarization effects due to an accumulation of charges underneath the injection contact we use a source of low activity (trigger rate $<100 \mathrm{~Hz}$ or $<5.6 \times 10^{3} \alpha / \mathrm{cm}^{2} \mathrm{~s}$ in the active area) and a short measurement duration of 5 min per voltage setting. The injected charge is small enough that the field under the contact essentially remains unaltered from its ohmic value and we measure space-charge-free (SCF) transient curves at all voltages as described in Ref. 9 with the addition of a nonconstant ohmic field. This is further supported by the fact that all transient curves rapidly decay to zero after the charge's arrival at the opposite electrode, as we will decribe in Sec. III. The presence or absence of polarization effects, commonly associated with, e.g., polycrystalline CVD diamonds, has been investigated through the time dependence of the measured transient curves. We compare transient curves recorded in the first minute after voltage and source were applied to transient curves recorded seven minutes later. Absolute voltage values and curve shapes recorded in the 1 st and 7 th minute agree to be better than $3 \%$. The nearly perfect agreement of transient curves leads us to conclude that polarization effects are avoided during our measurement.

The readout electronics is based on a single-stage current amplifier with a bandwidth of $2 \mathrm{GHz}$ (see Fig. 2). ${ }^{10}$ The amplifier circuit has been developed for a dedicated application of polycrystalline CVD diamond detectors as a highspeed beam monitor system. ${ }^{11}$ It provides a linear amplification of positive and negative input current signals and is tuned to suit the noise and speed requirements of CVD diamonds. Prior to our measurements its amplification and response were calibrated with external test pulses. We measure an amplification of $A=11.5$ and a rms noise on the output of the amplifier, while the detector was connected and biased, 


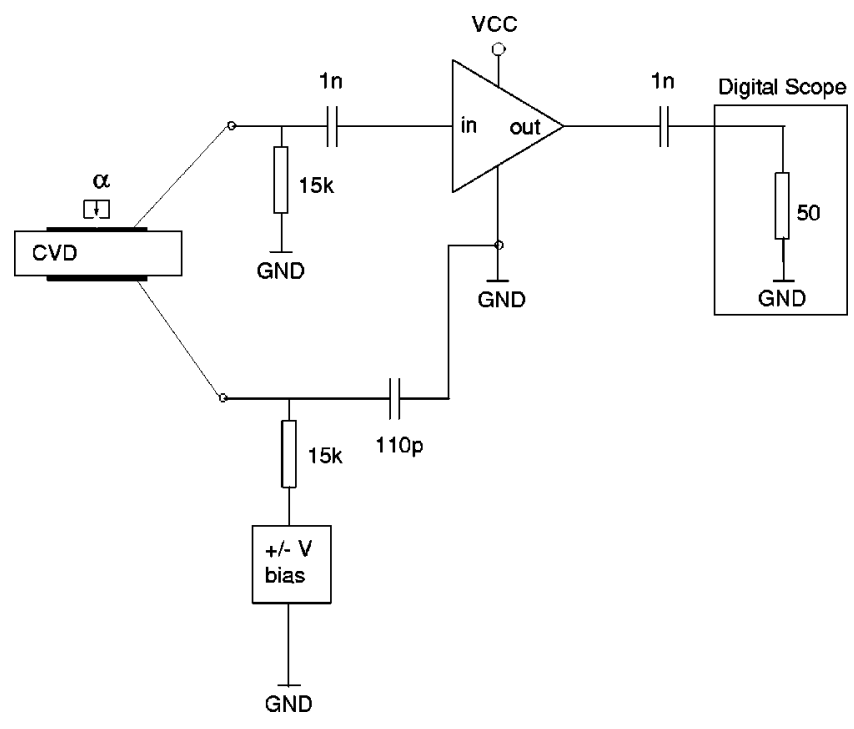

FIG. 2. Setup used for amplification of CVD diamond current signals.

of $\sigma=200 \mu \mathrm{V}$. The measurement precision of the absolute current amplitude is dominated by the uncertainty of the input resistance $R_{\text {in }}=45 \Omega$ and gain. The combined uncertainty is $\pm 15 \%$, while the repeatability of measurements is better than $\pm 5 \%$. The output of the current amplifier is sent via a 0.5-m-long signal cable to a LeCroy LC564A digital oscilloscope with an analog bandwidth of $1 \mathrm{GHz}$ and is digitized at $4 \mathrm{GS} / \mathrm{s}$.

\section{B. Data analysis}

Individual signals are recorded when the amplifier output amplitude exceeds a threshold of $0.7 \mathrm{mV}$ and a full width at half maximum (FWHM) pulse duration of larger than 2 ns. The requirement of a minimal pulse width was necessary to allow maximum sensitivity to small current pulses and suppress triggers on electronic noise. Care was taken to ensure that this trigger requirement rejects only electronic noise but is still significantly below the shortest observed diamond signals at any voltage setting. After individual signals are recorded for each bias voltage setting, they are offline corrected for trigger jitter and averaged to obtain an average pulse shape for each bias voltage setting. The measured voltage curve $U_{m}(t)$ is subsequently corrected for gain, input resistance, and the integration effect of detector capacitance and parallel stray capacitances at the input in order to obtain the current pulse as

$$
i_{m_{e, h}}(t)=\frac{1}{R_{\mathrm{in}} A}\left[R_{\mathrm{in}} C_{d} \frac{d U(t)}{d t}+U(t)\right]
$$

where $C_{d}=5 \mathrm{pF}$ denotes the sum of detector and stray capacitances at the input of the amplifier. ${ }^{4}$ For the determination of transit time, we fit the current pulse's rising and falling edges with complementary error functions, which yields the start and end times, $t_{s}$ and $t_{e}$, as the $50 \%$ point of the error function fits.

In order to verify our setup and analysis we carried out a reference measurement on a silicon diode using the identical setup and analysis technique later used for diamond. For the reference measurement we used a silicon $p-n$ diode supplied by SINTEF (Ref. 12) with a thickness of $1 \mathrm{~mm}$ and a full depletion voltage of $150 \mathrm{~V}$. The $\alpha$ source was mounted on top of the $p^{+}$-implant above a hole in the contact metallization and a positive reverse bias voltage was applied to the backplane, thus providing a measurement of the electron signal. From the current signal duration we determined an electron mobility in silicon of $1520 \mathrm{~cm}^{2} / \mathrm{V} \mathrm{s}$ at $200 \mathrm{~V}$. The electron drift velocity was measured in the voltage range of 200 $500 \mathrm{~V}$ and compares well to previously published data. ${ }^{13}$ Measuring the total charge by integrating the current signal yielded an ionization energy of $3.8 \pm 0.6 \mathrm{eV}$ for the creation of an electron-hole pair, which is in good agreement with the established value of $3.62 \mathrm{eV}$.

\section{ELECTRON AND HOLE CURRENT PULSES}

The measured hole and electron current pulse shapes are displayed in Fig. 3 for different settings of bias voltage $V$. Figure 3(a) shows the hole pulses resulting from negative values of $|V|=40,50,60,70,80,90,100,125,150,175,200$, $225,250,275,300,325,350$, and $375 \mathrm{~V}$ (from bottom to top of the graphs). Figure $3(\mathrm{~b})$ shows the electron pulses as a result of positive voltage settings of $|V|=70,85,100,125$,
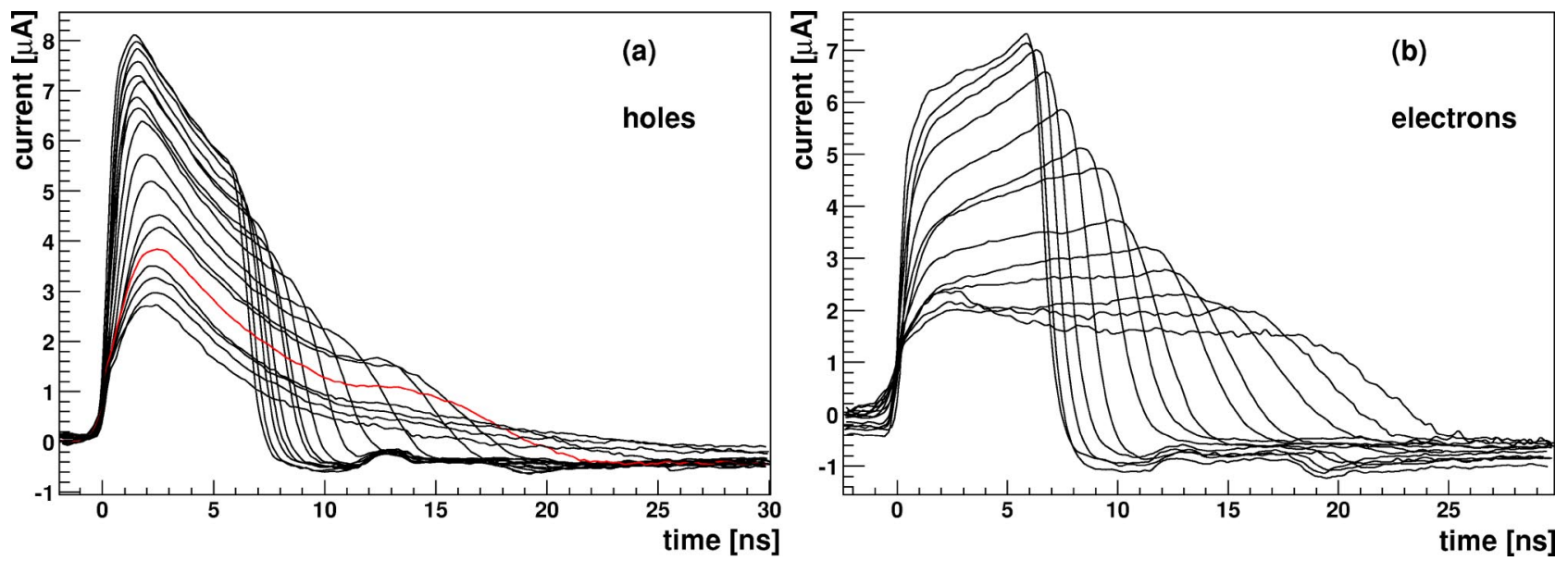

FIG. 3. Diamond current pulses for holes (a) with different negative bias voltages $(V<0)$ applied to the detector back contact and for electrons $(b)$ with different positive bias voltages $(V>0)$. 
$150,200,250,300,400,500,600,650$, and $690 \mathrm{~V}$ (from bottom to top of the graphs). The fast rising of the current signal is dominated by the electronic time constants of the amplification and readout system and remains approximately constant at higher voltages. The falling edge clearly shows the arrival of the charge cloud at the opposite electrode for electrons at all voltage settings and for holes at $|V|$ above 80 $\mathrm{V}$. We attribute the increased fall time at lower voltages to the increase in diffusion width as the drift time increases. Both the rising and falling edges are convoluted with the initial spatial distribution of the charge cloud although this constitutes a minor effect as can be seen from the pulse shapes at high voltages.

A significant decrease of current signal during the drift for holes and increase in current signal for electrons can be observed. While the decrease of the current amplitude for holes could naively be attributed to trapping of holes, there has to be another mechanism responsible for the increase of the current amplitude observed for the SCF transient for electrons. It will be shown in detail in Sec. VI that the convoluted effect of trapping of charge carriers with lifetime $\tau_{e, h}$ and of a negative space-charge concentration $N_{\text {eff }}$ in the bulk, leading to a nonconstant ohmic field, can explain the data reasonable quantitatively. For a linear electric field in the diamond bulk caused by a uniform negative space-charge concentration $N_{\text {eff }}$, it can be shown that the induced current follows an exponential behavior with two time constants in the drift region as

$$
\begin{aligned}
& i_{e, h}(t) \propto e^{t / \tau_{\mathrm{eff}}{ }_{e, h}-t / \tau_{e, h},} \\
& \tau_{\mathrm{eff}}=\frac{\epsilon \epsilon_{e, h}}{e_{0} \mu_{e, h}\left|N_{\mathrm{eff}}\right|} \approx \frac{\epsilon \epsilon_{0} t_{c} V}{e_{0} d^{2}\left|N_{\mathrm{eff}}\right|},
\end{aligned}
$$

where $\epsilon_{0}$ and $\epsilon$ denote the permittivity of vacuum and dielectric constant of diamond, $\mu_{e, h}$ the mobility of electrons and holes, $e_{0}$ the electron charge, and $t_{c}=t_{e}-t_{s}$ the transit time of charges through the diamond bulk. The first term in the exponent of Eq. (2) describes the exponential behavior of the current due to effective space charge (increasing current for electrons, decreasing current for holes); the second term accounts for the current's exponentional decrease as a result of charge trapping. In the case of holes the effect of negative space charge is enhanced by charge trapping, while in the case of electrons the decrease of amplitude due to trapping is more than compensated by the increase of current with time due to the increasing electric field in the space-charge region. In Secs. IV-VI we will determine the mobility, velocity, charge lifetime, and effective space-charge concentration using the information provided by the electron's and hole's current pulses.

\section{DRIFT MOBILITY AND VELOCITY}

The measurement of the charge cloud transit time $t_{c}$ provides a direct measurement of the average drift velocity as a function of the applied electrical field. For our measurement we calculate the average drift velocity as

$$
v_{\mathrm{dr}}(E)=d / t_{c} .
$$

The linear relation between drift velocity and electric-field strength as the actual definition of mobility is only valid at very low detector voltages. For further discussion we therefore introduce a formal calculated mobility as suggested in Ref. 14, which acts as an effective mobility $\mu^{*}$ at higher field strengths and includes effects of space charge. The calculation assumes that the field due to space charge present in our detector (see Sec. VI) is linear throughout the whole bulk. Consequently the effective mobility cannot be calculated from a linear relation between the applied drift voltage $V$ and the measured transit time $t_{c}$ as $\mu^{*}=d^{2} /\left(V t_{c}\right)$. We calculate an effective mobility $\mu_{e, h}^{*}$ from our measured transit time $t_{c}$ and detector voltage $V$ as

$$
\begin{aligned}
& \mu_{h}^{*}=-\frac{d^{2}}{2 t_{c} V_{c}} \ln \left(\frac{V+V_{c}}{V-V_{c}}\right), \\
& \mu_{e}^{*}=-\frac{d^{2}}{2 t_{c} V_{c}} \ln \left(\frac{V-V_{c}}{V+V_{c}}\right),
\end{aligned}
$$

where $V_{c}$ denotes the voltage necessary to compensate for the effect of space charge in the diamond bulk (for a detailed explanation of $V_{c}$ see Sec. VI). Although the formalism is similar to the full depletion voltage of silicon diodes, we do not a priori assume similar physical processes in diamond as present in $p-n$ silicon junctions, hence we explicitly avoid the term "full depletion voltage."

Figure 4(a) shows the measured drift velocity and effective mobility [Fig. 4(b)] as a function of externally applied drift field $|V| / d$. In these plots we only include measurements with applied voltages higher than $V_{c}$, i.e., voltages of $100 \mathrm{~V}$ or higher. We observe a significantly faster drift velocity for holes than for electrons. Both velocity curves clearly deviate from linear, and the electron and hole velocities start to saturate due to scattering of free carriers on optical phonons, in particular, in the field regime used for particle detectors $(0.8-1 \mathrm{~V} / \mu \mathrm{m})$. We measure for our sample an electron velocity of $5.6 \times 10^{6} \mathrm{~cm} / \mathrm{s}$ and a hole velocity of 7.5 $\times 10^{6} \mathrm{~cm} / \mathrm{s}$ at $0.8 \mathrm{~V} / \mu \mathrm{m}$.

Figure 4(a) illustrates that at voltages normally used for detector operation, the linear relation of drift velocity and field strength does not apply. In the regime where the drift velocity approaches the saturation velocity, it can be described instead as

$$
v_{\mathrm{dr}}=\frac{\mu_{0} E}{1+\frac{\mu_{0} E}{v_{s}}},
$$

where $\mu_{0}$ denotes the low-field mobility for electrons and holes, respectively, and $v_{s}$ their respective saturation velocities. ${ }^{15}$ The solid lines in Fig. 4(a) show the fits of Eq. (7) to the electron and hole data. The fits yield low-field mobilities $\mu_{0, e}=1714 \mathrm{~cm}^{2} / \mathrm{V} \mathrm{s}$ and $\mu_{0, h}=2064 \mathrm{~cm}^{2} / \mathrm{V} \mathrm{s}$ for electrons and holes, respectively, and saturation velocities of $v_{s, e}=9.6 \times 10^{6} \mathrm{~cm} / \mathrm{s}$ and $v_{s, h}=14.1 \times 10^{6} \mathrm{~cm} / \mathrm{s}$ for electrons and holes, respectively. For our sample those fit values pro- 

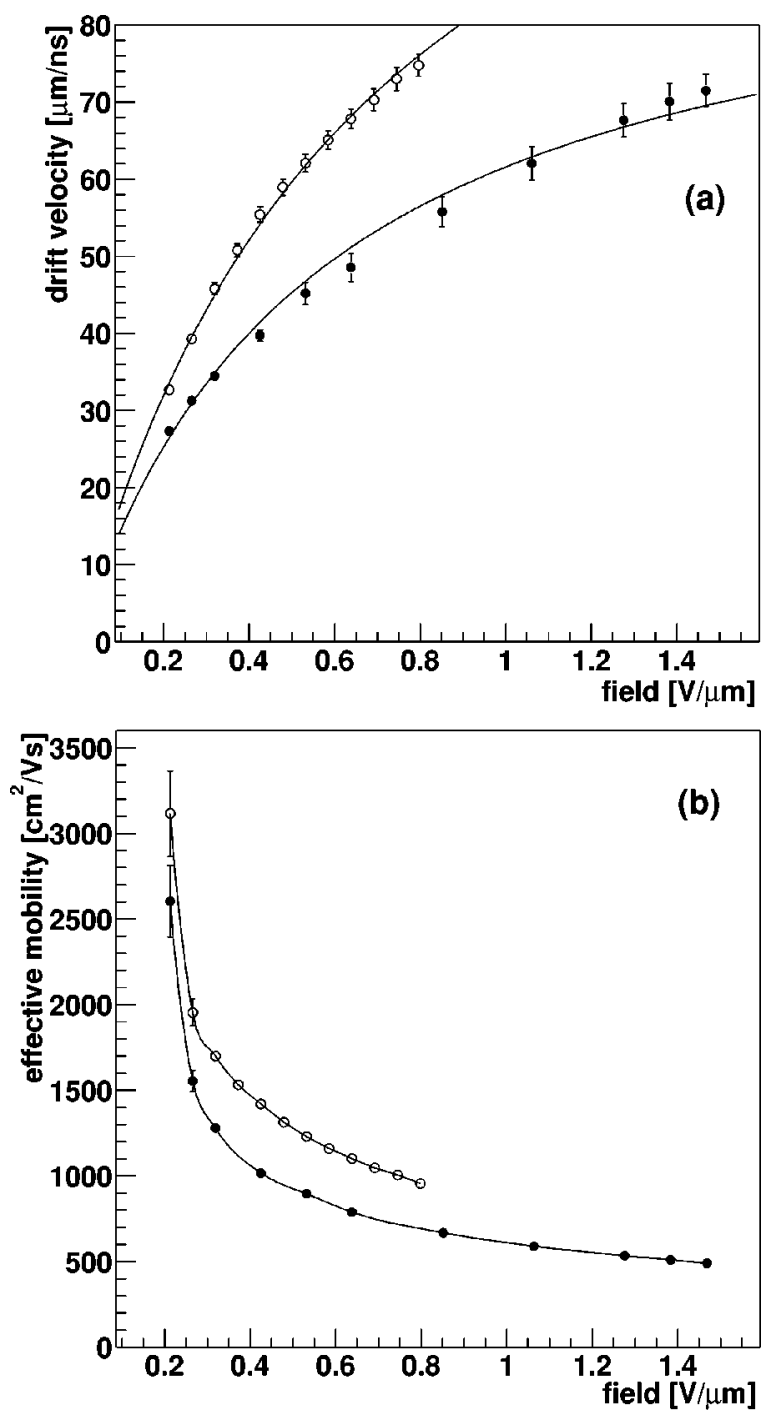

FIG. 4. Average drift velocity (a) and mobility (b) as a function of applied drift field $|V| / d$. The solid marker denote electrons and the open markers denote holes. The solid lines in (a) show fits of Eq. (7) to the data. The solid lines in (b) are only for visual guidance.

vide a good approximation of the drift velocity at fields used for detector operation.

The effective mobility shown in Fig. 4(b) reduces with increasing electrical field as the saturation velocity is approached. This reduction is generally observed in semiconductor materials, the dominant scattering mechanism being associated with optical phonons, and depends on factors such as the optical-phonon energy, relative trapping times of electrons and holes, and charge density. In applications such as diodes operated in the forward direction the voltage gradient across the material is desired to be as low as possible. Previous measurements under these conditions of low electric fields have reported mobilities as high as $4500 \mathrm{~cm}^{2} / \mathrm{V} \mathrm{s}$ (Ref. 6) on this type of diamond. The measurements reported here at low detector voltage, which are obtained with comparably low injected current density, independently verify previously measured mobility values for this type of diamond. ${ }^{6}$

Apart from optical-phonon scattering which leads to a reduction of mobility and velocity saturation, the measured drift velocity may also be limited by carrier-carrier scattering in the very early phases (approximately $1 \mathrm{~ns}$ ) of the drift. We estimate the possible systematic reduction of our measured velocities due to carrier-carrier scattering to be a maximum of $6 \%$ at the highest field strength data and less for lower field strength. For this estimate we used our measurement's peak carrier density and diffusion coefficients together with earlier published results on carrier-densityrelated reduction of drift velocity in diamond. ${ }^{16}$

\section{CHARGE-CARRIER LIFETIME}

During the drift process electrons and holes can be trapped which leads to a reduction of current signal if the charge-carrier lifetime is in the order of or below the transit time. Charge trapping results, for example, in a significant reduction of signal in polycrystalline CVD diamond. For the measurement of free-charge-carrier lifetimes for electrons and holes, we use the total induced charge through the integration of the measured current pulses at different voltages. In the following we use two methods for the determination of $\tau_{e}$ and $\tau_{h}$.

\section{A. Method of relative charge deficit}

We determine the total induced charge through the numerical integration of the measured current pulse as

$$
Q_{e, h}(V)=\int_{t_{s}}^{t_{e}} i_{m_{e, h}}(V, t) d t .
$$

In the case of a constant drift velocity, which is given by our average measured drift velocity, the relative charge deficit can be related to the charge-carrier lifetime using Eq. (2) as

$$
\frac{Q_{0_{e, h}}-Q_{e, h}(V)}{Q_{0_{e, h}}} \approx \frac{d}{2 \tau_{e, h}} \frac{1}{v_{\mathrm{dr}_{e, h}}},
$$

where $Q_{0_{e, h}}$ denotes the total charge in the absence of trapping.

We therefore use the dependence of $Q_{e, h}$ on the inverse drift velocity to determine the charge-carrier lifetime for electrons and holes. Figure 5 shows the total integrated charge as a function of the applied drift voltage for electrons and holes. Both curves show a clear saturation of the total charge above $|V|=200 \mathrm{~V}$. Electron and hole charges saturate at approximately the same values, which already indicates that there is no significant difference in electron and hole lifetimes in the range of the measured transit times.

Equation (9) assumes that a drift voltage is applied which is larger than the voltage required to compensate for negative space charge (see Sec. VI). We therefore use only measurements with $|V|>100 \mathrm{~V}$ to determine the relative charge deficit. The dependence of $Q_{e, h}$ on the inverse drift velocity is shown in Fig. 6 for electrons and holes. Linear fits to the graphs for electrons (solid line) and holes (dashed line) yield lifetimes of $\tau_{e}=33.8_{-6}^{+10} \mathrm{~ns}$ and $\tau_{h}=35.8_{-9}^{+20} \mathrm{~ns}$, respectively.

The extrapolation to infinite drift velocity, i.e., $1 / v_{\mathrm{dr}}=0$, allows us to estimate the generated ionization charge in the absence of trapping. The fit extrapolation yields a total ion- 


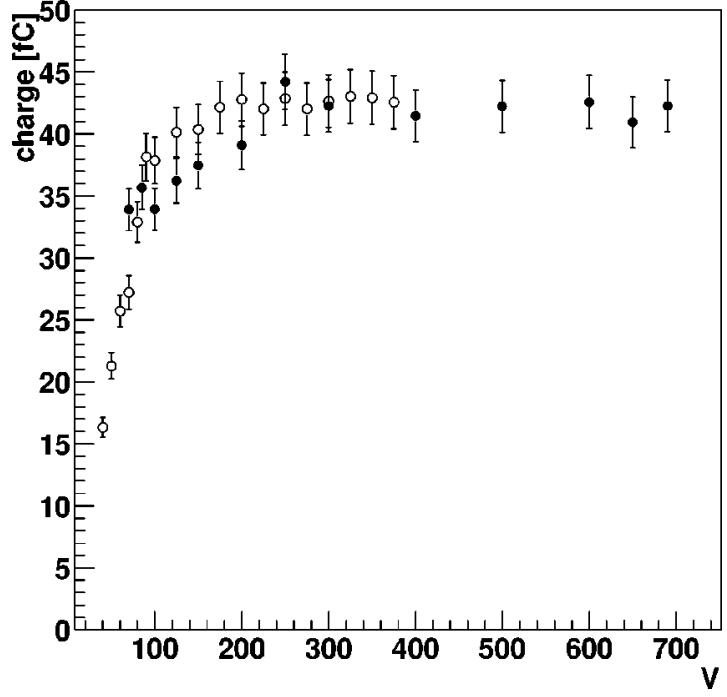

FIG. 5. Total induced charge for electrons (solid markers) and holes (open markers) as a function of applied drift voltage.

ization charge of $Q_{0_{e}}=47.6 \pm 1.6 \mathrm{fC}$ and $Q_{0_{h}}=47.5 \pm 2.2 \mathrm{fC}$ for electrons and holes, respectively. Including a correction for small base line shifts at the start and end of the pulse we measure a total ionization charge of $49 \mathrm{fC}$. Together with the calculated energy loss of $5.38 \mathrm{MeV}$ (Ref. 17) we estimate the energy for the creation of an electron-hole pair to be $17.6 \pm 2.7 \mathrm{eV}$, which is well in the range of previously reported values of $13-24 \mathrm{eV}$ for the ionization energy in diamond for $\alpha$ particles. ${ }^{16,18-20}$ Given the rather large $15 \%$ error on the absolute calibration of our amplifier, our measured value is in agreement with precision measurements of the ionization energy reporting values of $13.2-13.6 \mathrm{eV} .{ }^{19,21}$ It should be noted, however, that the absolute calibration of the system is not required for any analysis of results presented in this paper. The measurement of velocity, lifetime, and effective space charge only uses either the duration of the current pulse or its relative change of amplitude. For a better precision on the measurement of ionization energy it is preferable to calibrate the detector-electronic setup in an absolute way as it was done, for example, by Canali et al. ${ }^{19}$

\section{B. Method of charge correction}

From Eq. (2) it can be seen that the effect of charge trapping can be compensated, if $i_{m_{e, h}}(t)$ is multiplied by a

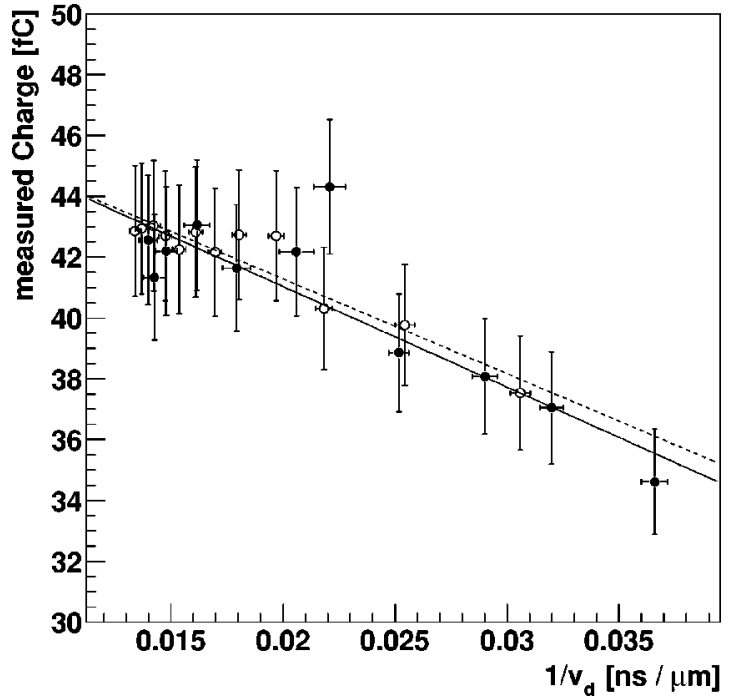

FIG. 6. Measured ionization charge for electrons (solid markers) and holes (open markers) as a function of inverse drift velocity.

factor $e^{+t / \tau_{\text {corr }}{ }^{4}}$ The resulting corrected current pulse replaces $i_{m}(t)$ in the current integration of Eq. (8). If the choice of $\tau_{\text {corr }}$ matches the actual charge-carrier lifetime, the total corrected charge $Q_{c_{e h}}$ will be a constant as a function of voltage for $|V|>100 \mathrm{~V}$. This behavior is illustrated in Fig. 7 for different choices of $\tau_{\text {corr }}$. From Fig. 7 it is obvious that the method becomes insensitive if the charge lifetime is much larger than the transit time, but that it is quite sensitive to a possible underestimation of the real lifetime. The fitted slopes at different values of $\tau_{\text {corr }}$ are further used to determine $\tau_{e}$ and $\tau_{h}$. The fitted slopes as a function of $\tau_{\text {corr }}$ are shown in Fig. 8 for electrons (solid markers) and holes (open markers). The horizontal dashed lines mark the $\pm 1 \sigma$ accuracy of the slope fits. On a $+3 \sigma$ level of accuracy our measurements are consistent with lifetimes in the microsecond range being reported in Ref. 6. From the zero crossing of the graph we extract the charge lifetime for electrons and holes as $\tau_{e, h}=40_{-10}^{+25} \mathrm{~ns}$.

Both methods yield consistent results for electron and hole lifetimes. The measured lifetimes for this sample of single-crystal CVD diamond significantly exceed the transit times of drifting charges and are above several tens of nanoseconds. This constitutes a dramatic improvement over previously reported charge lifetimes in high-quality polycrystalline CVD diamonds. ${ }^{22}$
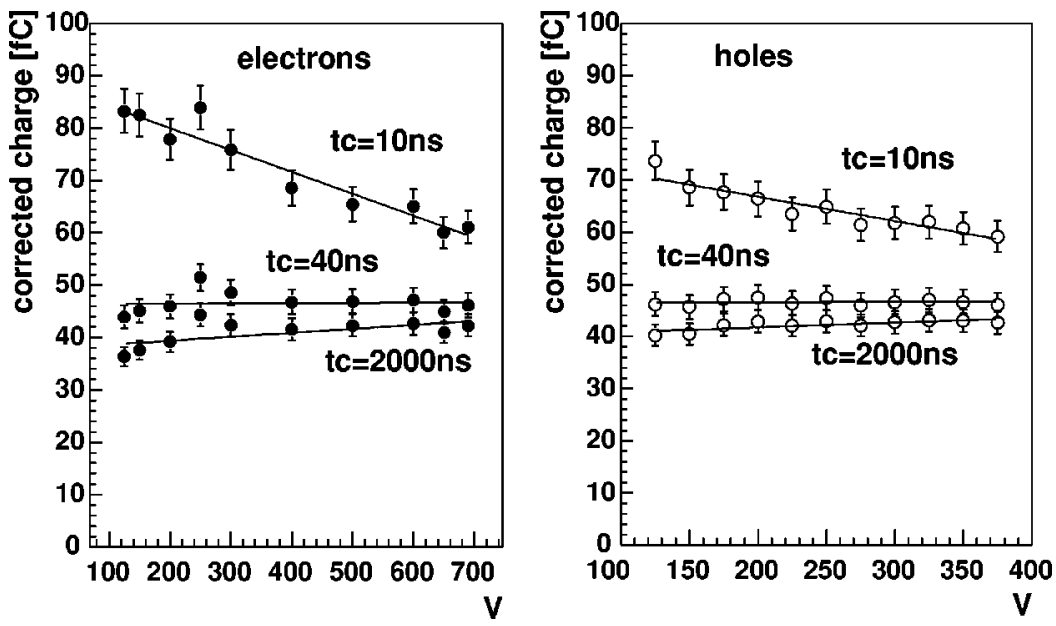

FIG. 7. Corrected charge as a function of drift voltage for electrons (a) and holes (b) with different choices of $\tau_{\text {corr. }}$ 


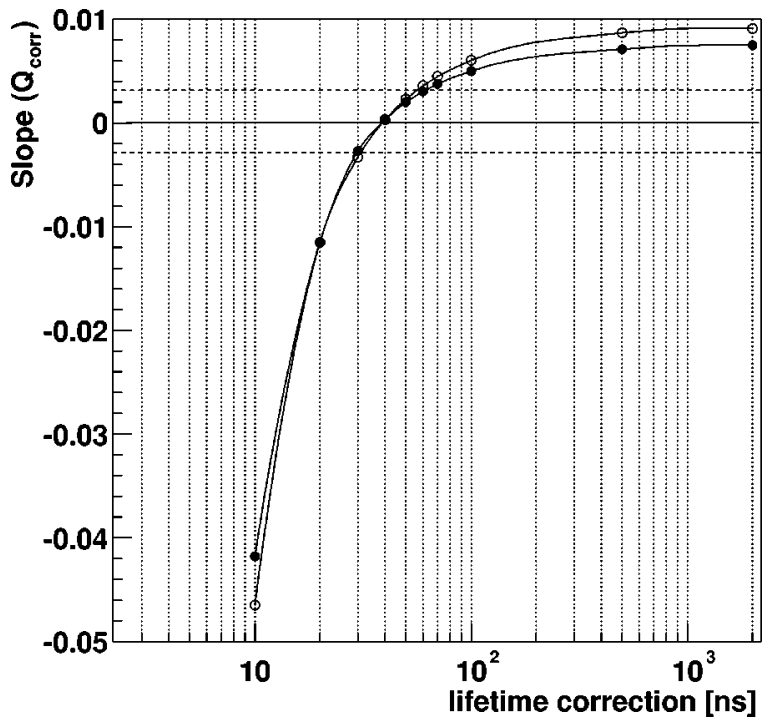

FIG. 8. Slope of $Q_{c_{e, h}}(V)$ as a function of $\tau_{\text {corr }}$.

\section{NET EFFECTIVE SPACE CHARGE}

The exponential shape of electron and hole current pulses in Fig. 3 can be explained by a net effective space charge in the diamond bulk. In the simpliest case of a uniform effective space charge, this leads to a linear dependence of the electric field as a function of position in the bulk. This in turn causes an exponential increase (electrons) or decrease (holes) of the induced current.

\section{A. Determination of average effective space charge}

Using the simple case of a uniform effective space charge we can determine an average effective space charge in the diamond bulk. The assumption of an effective space charge in the diamond bulk is supported by the approximately exponential behavior of the current pulses. From the relative sign of increase/decrease for electrons and holes we can determine that, although the space charge can be caused by more than one type of defect, the cumulative effect of those defects is that of a negative space charge.

Figure 9 shows the behavior of the hole signal as the voltage $|V|$ is increased. In this configuration the high electric-field region is on the side where the charge is injected, which is shown schematically in the insert of Fig. 9. As the width $w$ of the bulk with $E_{d} \neq 0$ increases proportional to $\sqrt{V}$, the increasing drift path and field leads to an increase of induced signal. When a voltage $V_{c}$ is reached, which corresponds to the voltage necessary to just compensate for the negative space charge, the hole pulse starts to exhibit the falling signal edge which is characteristic for the arrival of the charge cloud at the opposite electrode. The first sign of this charge cloud arrival appears at $V=-70 \mathrm{~V}$ and is clearly present at $V=-80 \mathrm{~V}$. This interpretation is also consistent with the observation that we could not detect any electron signal for voltages below $70 \mathrm{~V}$, as the $\alpha$ particles are injected into a field-free region for positive voltages below $70 \mathrm{~V}$. The appearance of a falling signal edge in the hole pulse may serve as an indication for $V_{c}$ but is not sufficiently accurate

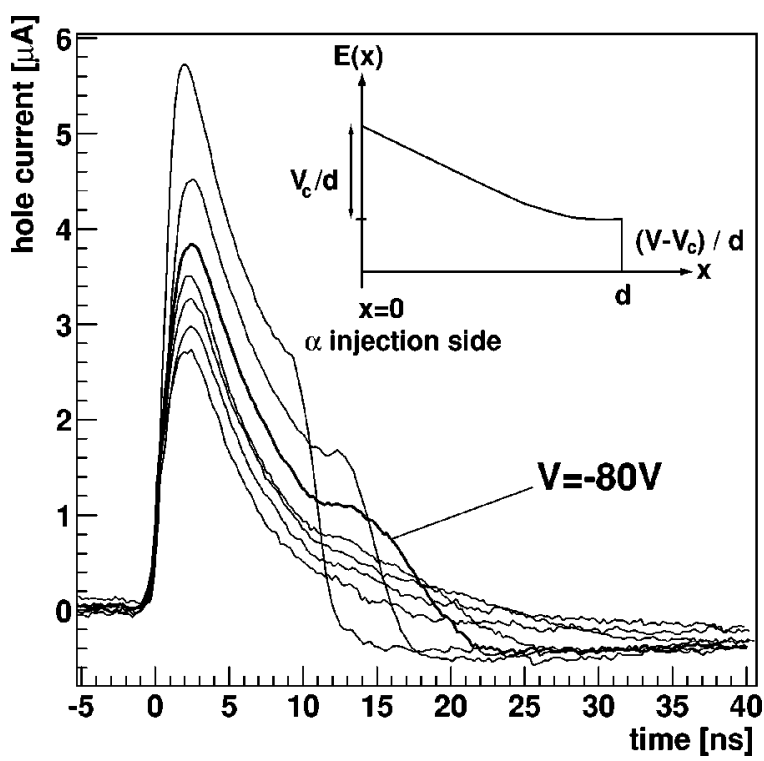

FIG. 9. Hole pulses for $V=-40,-50,-60,-70,-80,-90$, and $-100 \mathrm{~V}$ (bottom to top amplitude). The insert shows a schematic representation of a possible electric field with $V>V_{c}$.

for a precise determination of $V_{c}$ as it depends on the spatial distribution of initial charge deposition and diffusion.

For an accurate determination of $V_{c}$ we use the total integrated charge, corrected for charge trapping, as introduced in Sec. V as

$$
Q_{c_{h}}(V)=\int_{t_{s}}^{t_{e}} i_{m_{h}}(V, t) e^{+t / \tau_{h}} d t,
$$

$$
N_{\text {eff }}=\frac{2 \epsilon \epsilon_{0} V_{c}}{e_{0} d^{2}},
$$

The second equation gives the relation between $N_{\text {eff }}$ and $V_{c}$ for a uniform effective space-charge concentration.

Figure 10 shows the dependence of $Q_{c_{h}}$ on $\sqrt{V}$. The plot

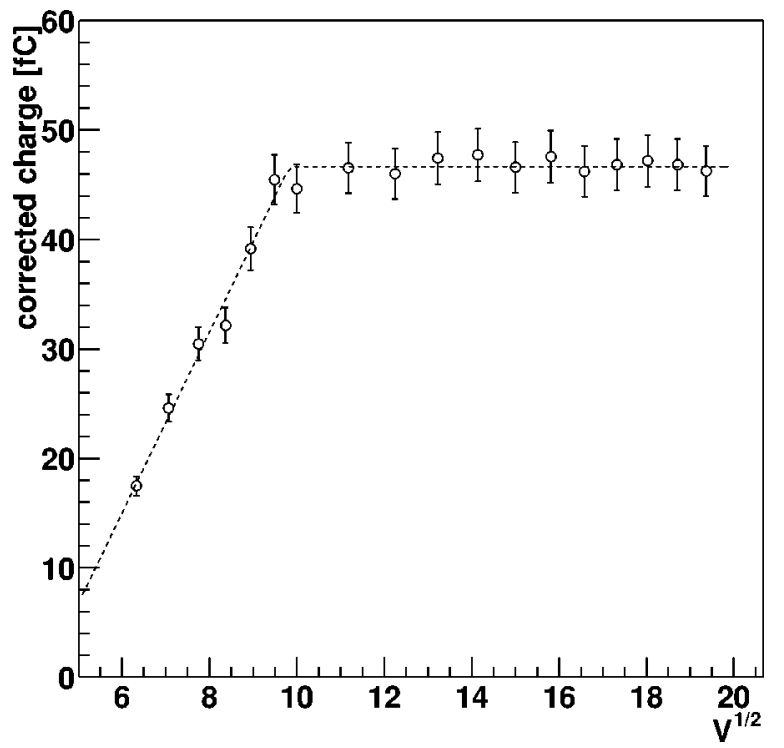

FIG. 10. Total integrated hole charge as a function of $\sqrt{V}$. The dashed line indicates the fit to determine $V_{c}$. 

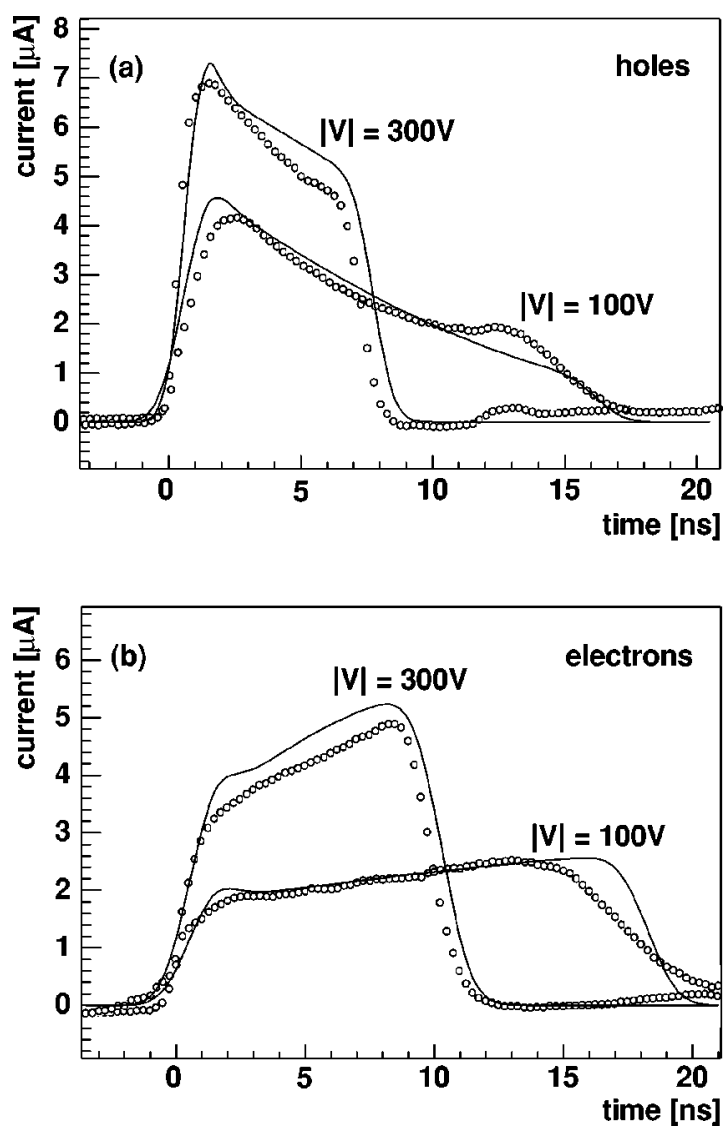

FIG. 11. Base line corrected measured current pulses (markers) and simulated current pulses (lines) for holes (plot a) and electrons (plot b).

shows a clear kink when $V$ reaches $V_{c}$ and remains constant as expected for higher voltages. From a fit to the graph (shown as dashed line) we determine $V_{c}=96 \mathrm{~V}$ and an average effective negative space-charge concentration of $N_{\text {eff }}$ $=2.8 \times 10^{11} \mathrm{~cm}^{-3}$. From the linear dependence on $\sqrt{V}$ for $V$ $<V_{c}$ we can also conclude that the effective charge collecting electrode remains the physical electrode for a CVD diamond detector due to the high resistivity of the electrically neutral fraction of the bulk.

\section{B. Further considerations on $\boldsymbol{N}_{\text {eff }}$}

While the above assumption of a uniform effective space charge and linear field is a good approximation for higher detector voltages, we observe a deviation from linear fields at detector voltages around and below $V_{c}$. Electron and hole current pulses at $|V| \approx V_{c}$ show a nearly flat behavior towards the end of the drift time as shown in the $100-\mathrm{V}$ measurement data of Fig. 11 for drift times larger than $10 \mathrm{~ns}$. This suggests that at low detector voltages the electric field starts to deviate from its linear dependence through the bulk and becomes approximately constant towards the charge collecting electrode. This is also evident in the extrapolation of the linear fit in Fig. 10 to the horizontal axis. In the linear extrapolation $Q_{c_{h}}=0$ is reached for an applied voltage of $V \approx 25 \mathrm{~V}$ rather than at $V=0 \mathrm{~V}$. At this low voltage the effective space charge may significantly deviate from the above measured value of $N_{\text {eff }}$ and may in turn depend, e.g., on the combination of generation current and trapping center density, which can lead to a nonlinear field distribution and extended field-free regions in the bulk. ${ }^{23}$ Further investigations are currently underway to study the diamond behavior and field distribution through TCT measurements at very low voltages using improved electronics.

Another observation concerns the current increase during the drift time observed for electrons. The current grows faster for higher detector voltages, which indicates that the effective space charge, though nearly uniform, depends on the detector voltage. This is not the case for holes. To illustrate this behavior qualitatively we compare our data to a current pulse simulation in Fig. 11. The simulation assumes a uniformly distributed negative space charge and our measured values for charge lifetime and drift velocity as a function of electric field. Figure 11 compares the measured base line corrected current pulses for holes and electrons to the simulation at two different voltages: at $100 \mathrm{~V}$ close to $V_{c}$ and at a typical detector operation voltage $(300 \mathrm{~V})$. For holes (plot a) we assumed the same effective space charge of 2 $\times 10^{11} \mathrm{~cm}^{-3}$ in the simulation at both voltages and the simulation agrees well to the data for pulse duration, amplitude, and current slope during the drift. For electrons (plot b) we achieved a good agreement, in particular, modeling the current slope during charge drift, only when using different values for the effective space charge at $100 \mathrm{~V}$ (simulation assumes $0.8 \times 10^{11} \mathrm{~cm}^{-3}$ ) and $300 \mathrm{~V}$ (simulation assumes 5 $\times 10^{11} \mathrm{~cm}^{-3}$ ). All other diamond parameters are kept constant for all simulations. One possible origin of this behavior may be the combination of current injection through the contacts in combination with rather uniformly distributed charge trapping centers, which can result in a uniform but voltagedependent space-charge density. This leads us to expect a sample dependence of this behavior as well as a dependence of the surface preparation process. Further investigations are currently being prepared to study the dependence on the surface preparation as well as intrinsic sample-to-sample variations.

\section{CONCLUSION}

We used the transient-current technique (TCT) to measure the transport properties of free charge carriers in chemical-vapor deposition (CVD) diamond. The use of a fast low-noise RF amplifier allowed us to directly measure the induced current for electrons and holes, which were generated by an $\alpha$ source. We used this method to study the electron and hole transport properties in a sample of singlecrystal CVD diamond under conditions usually used for particle detectors. We measured the drift velocity and mobility as a function of electric field at room temperature. In the high-field regime typical for particle detectors we obtain drift velocities of $5.6 \times 10^{6} \mathrm{~cm} / \mathrm{s}$ and $7.5 \times 10^{6} \mathrm{~cm} / \mathrm{s}$ for electrons and holes at $E=0.8 \mathrm{~V} / \mu \mathrm{m}$, respectively, and saturation velocities of $v_{s, e}=9.6 \times 10^{6} \mathrm{~cm} / \mathrm{s}$ and $v_{s, h}=14.1 \times 10^{6} \mathrm{~cm} / \mathrm{s}$ for electrons and holes, respectively. The charge-carrier lifetime was measured to be $\tau_{e, h}=40_{-10}^{+25} \mathrm{~ns}$. This charge lifetime is significantly longer than the charge transit time, which shows that charge trapping is no longer limiting the obtainable signal in the highest-quality single-crystal CVD diamond. The 
behavior of the induced current indicates the presence of a negative effective space charge in the diamond bulk for our sample. For the tested sample we obtained an average effective negative space charge of $N_{\text {eff }}=2.8 \times 10^{11} \mathrm{~cm}^{3}$ with indications for a dependence on detector voltage.

Our results obtained on a single-crystal CVD diamond show the excellent sensitivity of the TCT method to fundamental charge-carrier properties. The method allows one to characterize several of those parameters with a single direct measurement. While the scope of this work was to demonstrate the characterization of a single-crystal CVD diamond with the transient-current technique using $\alpha$ particles, we will continue our investigation to study sample-to-sample variations of transport properties and their dependence on the CVD fabrication process.

\section{ACKNOWLEDGMENTS}

The authors want to thank Professor Marko Mikuz and Dr. Gregor Kramberger of the Institute Jozef Stefan, Ljubljana, Slovenia for their support and critical reading of the manuscript. Their experience with using the transient-current technique has been vital for the development of the analysis procedure used in this paper.

${ }^{1}$ M. Lampert and P. Mark (Academic, New York, 1970).

${ }^{2}$ V. Eremin N. Strokan, E. Verbitskaya, and Z. Li, Nucl. Instrum. Methods Phys. Res. A 372, 388 (1996).

${ }^{3}$ Z. Li, C. J. Li, V. Eremin, and E. Verbitskaya, Nucl. Instrum. Methods Phys. Res. A 388, 297 (1997).

${ }^{4}$ G. Kramberger, Ph.D. thesis, University of Ljubljana, 2001.
${ }^{5}$ The RD42 Collaboration, R\&D Proposal, Development of Diamond Tracking Detectors for High Luminosity Experiments at LHC, DRDC/ P56, CERN/DRDC 94-21, May 1994.

${ }^{6}$ J. Isberg, J. Hammersberg, E. Johansson, T. Wikström, D. J. Twitchen, A. J. Whitehead, S. E. Coe, and G. A. Scarsbrook, Science 297, 1670 (2002).

${ }^{7}$ Element Six Ltd., King's Ride Park, Ascot, Berkshire SL5 8BP, UK.

${ }^{8}$ CERN status report of the RD42 collaboration to LHCC, CERN, 30 January 2004.

${ }^{9}$ M. Lampert and P. Mark (Academic, New York, 1970), Sec. 6.1.1, pp. $115-119$.

${ }^{10}$ FOTEC, Viktor Kaplan Str. 2, A-2700 Wiener Neustadt, Austria.

${ }^{11}$ H. Frais-Kölbl, E. Griesmayer, H. Kagan, and H. Pernegger, IEEE Trans. Nucl. Sci. 51, 3833 (2004).

${ }^{12}$ SINTEF Electronics and Cybernetics, Strindveien 4, 7465 Trondheim, Norway.

${ }^{13}$ C. Jacoboni, C. Canali, G. Ottaviani, and A. Alberigi Quaranta, SolidState Electron. 20, 77 (1977).

${ }^{14}$ G. Lutz, Semiconductor Radiation Detectors (Springer, Berlin, 1999), pp. $102-105$.

${ }^{15}$ Z. Li and H. W. Kraner, Nucl. Phys. B 32, 398 (1993).

${ }^{16}$ L. S. Pan, D. R. Kania, P. Pianetta, and O. L. Landen, Appl. Phys. Lett. 57, 623 (1990).

${ }^{17}$ M. J. Berger, J. S. Coursey, and M. A. Zucker, ESTAR, PSTAR, and ASTAR: Computer Programs for Calculating Stopping-Power and Range Tables for Electrons, Protons, and Helium Ions (version 1.2.2), http:// physics.nist.gov/Star (2004, January 17), National Institute of Standards and Technology, Gaithersburg, MD.

${ }^{18}$ L. S. Pan et al., J. Appl. Phys. 74(2), 1086 (1993).

${ }^{19}$ C. Canali, E. Gatti, S. F. Kozlov, P. F. Manfredi, C. Manfredotti, F. Nava, and A. Quirini, Nucl. Instrum. Methods 160, 73 (1979).

${ }^{20}$ P. J. Dean and J. C. Male, J. Phys. Chem. Solids 25, 311 (1964).

${ }^{21}$ L. S. Pan, S. Han, and D. R. Kania, Diamond: Electronic Properties and Applications (Kluwer Academic, Dordrecht, 1995).

${ }^{22}$ J. Hammersberg, J. Isberg, E. Johansson, T. Lundström, O Hjortstam, and H. Bernhoff, Diamond Relat. Mater. 10, 574 (2001).

${ }^{23}$ V. Eremin, E Verbitskaya, and Z. Li, Nucl. Instrum. Methods Phys. Res. A 476, 556 (2002). 